E3S Web of Conferences 2, 01001 (2014)

DOI: $10.1051 / \mathrm{e} 3$ sconf/ 20140201001

(C) Owned by the authors, published by EDP Sciences, 2014

\title{
Science and the Future: Introduction
}

\author{
Angelo Tartaglia ${ }^{1,2, a}$ \\ ${ }^{1}$ Department of Applied Science and Technology, Politecnico di Torino, Corso duca degli Abruzzi 24, 10129, \\ Torino, Italy \\ ${ }^{2}$ INFN, Via Pietro Giuria 1, 10126, Torino, Italy
}

\begin{abstract}
The contradiction between physical and economical sciences concerning the growth of the production/consumption mechanism is analyzed. It is then shown that if one wishes to keep the security level stable or to enhance it in a growing economy the cost of security grows faster than the gross wealth. The result is a typical evolution in which the net wealth increases up to a maximum, then abruptly collapses. Besides this, any system of relations based on a growing volume of exchanges is bound to go progressively out of control.

The voluntary blindness of the ruling classes toward these facts is leading our societies to a disaster. This fate is not inescapable provided we learn to dismantle the myth of perpetual growth.
\end{abstract}

\section{Foreword}

The history of the human kind on the planet Earth is extremely short as compared to geological or cosmic times, but is felt as being already quite long in the human perception. In fact the part of our common past properly considered as historical has had a duration of approximately 400 generations: nothing, in a world where every year some 125 million new human beings are born (more than 343,000 per day). Even in such a short time span the impact of humanity on Earth, never irrelevant (as some of the talks in these conference will show), has undergone a sharp raise starting from some fifteen generations ago. The birth and development of modern experimental science (mainly physics in all specifications) spurred technology, in a previously unthinkable way. These new powerful tools have been made available to a species defining itself as "intelligent"; a species whose behaviour has at all times been conformed to non-scientific criteria and directed to not properly rational ends. The de facto behaviour of the human beings has however ever since been covered, like by a coat or a sort of Grecian theater mask, by a conceptual framework intended to justify it or even motivate it ex post. Any human society, since Sumerian times up to the present day, has worked out an ideological self-representation that "explains" it is right that it happens precisely what happens.

\section{The role of science and the problem of limits}

The development of the scientific knowledge and method has progressively brought to evidence some inconsistencies or plain contradictions between ideological visions and the material advancement of

\footnotetext{
ae-mail: angelo.tartaglia@polito.it
} 


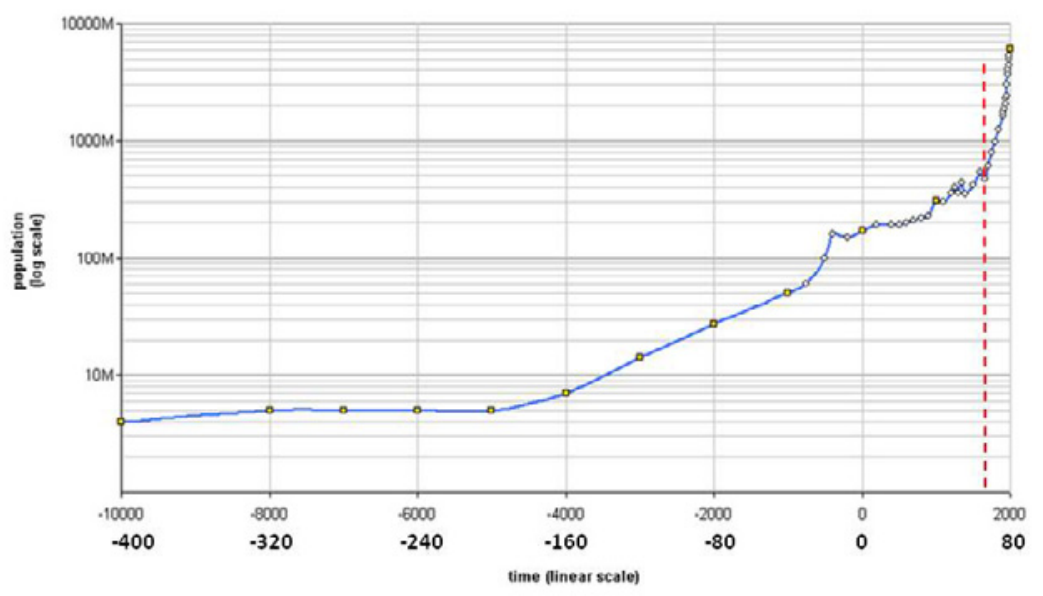

Figure 1. Growth of the world population in time; the lower line in the abscissae measures time in conventional human generations ( 25 years). The dashed line marks the date (some 14 generations ago) from which the modern "explosion" of the population starts, accompanying the industrial revolution.

human societies. These inconsistencies and contradictions have in general to do with the concept of limit, meant as material constraint. In ideological views, different and changing in time as they are, the very idea of "limit" has never been popular: one meets sheer refusal (we shall see an interesting example tomorrow evening in the "Last call" movie) or, at times, the recognition that 'well, material constraints do exist, but they are exceedingly far from our present condition, so that they are and must be uninfluential on the decisions we are called to assume here and now'.

Another way to describe this attitude could be that mankind tends and pretends to live exclusively in the present, removing any concern about a non-immediate future. In short, a time dimension with a thickness close to zero. This behaviour is what we currently expect from non-human animal species; the point however is that the human being, especially after the onset of modern science, has got tools and methods enabling to look, in a reasonably reliable way, even into a non-immediate future. What can be seen in that, scientifically fathomable, future are developments contradicting the perspectives advertised by ideology (here I am not referring to any peculiar political vision, rather to the most common way of thinking of humans and in particular of the members of the "ruling class").

The importance and inescapability of material constraints is indeed rather obvious and has been perceived as such all along the history of human thought. That awareness, however, has in general not exerted any real and effective influence on actions. After the start of the industrial revolution we find an example of such awareness in the positions of rev. Malthus, who apparently concentrated on the demographic issue only. Only in very recent times, however, "exact" sciences have tackled the problem of constraints: general public was involved in 1972, when The limits to growth was published. 
We shall recall that event tomorrow afternoon. The report, produced by a team of the MIT (Donella Meadows, Dennis Meadows, Jørgen Randers, William Behrens III) and commissioned by the Club of Rome, was promoted by Aurelio Peccei, a native of the city where we are now.

The Limits to Growth was immediately attacked (ideologically) both from the "left" and from the "right": the idea that material growth of the economy could have physical limits was unacceptable. This pretended "unacceptability" is related to a sort of human grandiose delusion that should be matter for psychologists, but is in fact deeply rooted in a culture, especially European, now spread worldwide by right of conquest, which maintains that matter is subordinate to "spirit". "Spirit" here is not the one of religion, rather, if anything, the one of Hegelianism; but I neither can nor want to enter philosophical considerations, so let me drop this issue.

It's a given that matter: a) exists; b) evolves according to laws which depend neither on philosophical nor on economical schools and are not at all influenced by political affairs. Be they acceptable or not, no parliament can reform the physical laws and they are not even listed in the stock market.

Since 1972 to the present day a true scientific literature has been produced concerning the material constraints human societies cannot avoid to come to terms with, even though they are not willing to do so. A trivial search on an academic search engine pinpoints in a split second more than 2,000,000 scientific papers on the climate change issue, produced in universities and research institutions. If you try a query on the peak oil you find not less than 1,000,000 articles, and so on. For comparison, a subject like superconductivity gives less than 650,000 answers and a general term, typical of my normal research activity, like cosmology produces less than 500,000 titles.

National and international conferences have grown in number and frequency and appeals have been proposed to reroute the world economy and to curb the mythical "growth". A prestigious example has been the Stockholm memorandum, signed in 2011 by 18 Nobel laureates. Positive intentions have been expressed hither and thither; some states have signed agreements such as the Kyoto protocol of 1997, or have defined targets to be pursued, like the 20-20-20 directive of the European Union; and so on. Something has started to move and is moving. In substance, however, the problem has not been tackled in its root and the global situation has worsened; in the best case one worries about the symptoms, not about the disease.

\section{Economics}

What I referred to above, when writing of the boom of specialized literature, were the sciences of nature, all more or less "exact", provided this attribute makes sense. Those sciences deal with material quantities and measure in units like kilograms and Joules (or $\mathrm{kWh}$ ). In that context not anything concerning the future is clear and unambiguous; there are uncertain and even controversial issues. The bases, however, and the rules of the game are definitely certain: the discussion may be about technical problems and ultimately on details, even if non-marginal ones (we shall see in the next days of the conference), but the substance is unique and clear.

But not only the sciences of nature exist; other disciplines can be qualified as sciences too, because of the method and accuracy in analysis and application. In particular, in the matter that we shall be discussing in these days a fundamental role is played by economics. A layman, as I am on this respect, is led to say that the object of economy as a science is human behaviour, like in a sort of social psychology applied to exchange relationships of goods and services within a given society; I should include also the production of goods and services directed, in a way or another, to the exchange. If it is really so (I beg the pardon of economists) analyses, "laws", mathematical formalization are necessarily statistical and the observations are mainly empirical, axioms are quite different from those of physics or even more of mathematics. In economics basic hypotheses, more than axioms, betray 
also the (human) world views typical of one or another society and lack the logical cogency of the axioms of natural sciences.

Having said that, economics too has enormously grown in complexity, internal differentiation and sophistication of the technical tools it uses. Economics is formulated in refined mathematical terms, manipulates quantitative data and produces numerical predictions. Of course a person like me, with a physicist's (and engineer's) mentality, gets puzzled looking at the numbers appearing in newspapers, even in the pages dedicated to economy and finance; not because of the values in themselves, but rather because of the systematic absence of anything like an uncertainty interval or, when modelling is used (i.e. almost always), of a confidence interval for predictions: we shall discuss this aspect on Thursday. My perplexity, as I have said, concerns communication through the media, but that is the channel which conveys messages to the general public.

Of course economy deals with material quantities, the ones measured in $\mathrm{kg}, \mathrm{kWh}$ and so on, but the most important quantity is in the end money; at least, everything ends being expressed in monetary terms. Now, again, a physicist cannot help but remark that money is fundamentally a conventional quantity, that expresses, within a given society, the distribution of the buying power (to a great extent coinciding with power, without further specifications), i.e. of the right of access to goods and services globally produced. In other words, there is an objective poverty, consisting in the scarcity or material inaccessibility of some good; then there is a "social" poverty depending on the unequal distribution of the "access titles". In our globalized world the difference between the two forms is more and more evanescent. In conclusion, though it appears to be paradoxical, the poor is poor because "he agrees to be poor" even though he does not know: he partakes in a giant Monopoly game whose rules he, more or less consciously, accepts. All this has nothing to do with the laws of thermodynamics, with the conservation of matter and energy, with climatology, with the laws of electromagnetism, and so on. Disparities and the fair or unjust distribution of the "access titles" is a match internal to mankind, but that match is played on a material stage; the rules of the game are, with more or less difficulty, pliable, the playing ground and its constraints not at all.

Economical science, by its nature, has to deal with both the material context and the internal affairs of human societies. It seems however, at least to an inexperienced person as I am, that the attention is most often focussed on human dynamics rather than on the material background. I shall fetch an image from a field very distant from economy, but much more familiar to me. Einstein succeeded in describing the gravitational interaction by a very famous (among specialists, of course) equation of which he used to say that the two sides (linked by the $=$ sign) were quite different: one was "marble", and it was the one rigorously and exclusively cast in mathematical and in particular in geometrical terms; the other was "wooden", and it was the one containing matter and energy, both entities expressed in mathematical forms without being mathematical in nature. In an entirely different area and by pure analogy I think I can say the proper domain of economy too has two sides: one "marble", which is the side of material bases; the other "wooden", which is the side of social dynamics. My feeling (and may be this conference will help to discuss the issue and make me change my mind), is that very often economy mistakes "marble" for "wood", without, by that, "marble" ceasing to be what it is.

\section{The problem of growth}

On the background described so far we find the prickly problem of "growth". "Growth", at first sight, is the most often used word on the press, by politicians, by economists when the discussion is about the world "crisis", started in 2008 and not yet ended, and about the way out from it. On the nature of that "crisis" I think this conference will express peculiar viewpoints; for the time being let us limit ourselves to the question: growth of what? 
In the debate among social parts and in the journalistic and popular language the growth people want to start up again is of consumption, of jobs (of consumption in order to make jobs grow), of investments, of production, of the "confidence" of "markets" and so on. When we come to the numbers, all that is recapped and summarized in one single parameter: the Gross Domestic Product, in short GDP. The latter, in the popularization of the media, is not even expressed by an absolute value, but only as an annual percent growth (lately even decrease): "GDP is at $\mathrm{x} \%$ ". Literally speaking this is nonsense; for the majority it means that things are good or bad, without worrying about why they are good or bad. Long since there is an active debate on the reliability of the Gross Domestic Product as an indicator of the socioeconomic wellness and prof. Giovannini could tell us much about the subject; as a matter of fact people goes on using GDP.

As I understand it, and I am a layman, GDP measures the volume of material and immaterial exchanges within a given economic system, provided they have a monetary value. That is the quantity whose growth everybody invokes. Stated in these terms one may have the impression that the issue is essentially an internal affair of human societies, all the more so, since, as I have said, the exchange can also be immaterial: a violin lesson contributes to the formation of the GDP, provided it is paid for (what is free of charge, in this context, besides not been counted, is at the least an extravagance).

Beyond the political and journalistic language, I can guess that the theoretical framework that includes the growth, rests on two postulates.

- a) The vitality of any human society can last in time if there are disparities among its members: in an electric circuit no current circulates if the potential is the same everywhere.

- b) Economic disparities, however, have drawbacks, dangerous for social stability. If you want to find a solution to these drawbacks or at least keep their effects under control, the volume of the economic exchanges must keep on growing: some would say that in order to maintain social differences, without producing lacerations, the global "wealth" is bound to a perpetual growth.

Is this the essence of an amateur theory of growth? Maybe.

However the problem is that any "exchange", including the apparently immaterial ones, has unavoidably a material base. By consequence the growth of the Gross Domestic Product is necessarily and in any case a growth of the amount of matter manipulated and transformed every year and an increase in the number of transformations to which even a fixed amount of matter is subject every year (at least part of the present audience has probably caught here a reference to the growth of the energy demand). When we speak of matter and its transformations we face rules of the game on which we have no control; they are, as I have already reminded, totally insensitive to political and even social affairs, to the spread, to the stock market quotations, as well as to the unemployment rate. At this stage the contradiction becomes manifest and, it is to be said, on one side we may heap opinions, on the other we find very stubborn facts.

There are three ways to manage these contradictions by the supporters of growth:

- reject the problem;

- recognize that the contradiction exists, but maintain that its practical effects are delayed to back of beyond future;

- propose a socioeconomic system where the material base remains constant and the GDP increases in its immaterial part only.

No doubt that the first "solution" is the most widespread. It includes those people who look at the issue of growth as at a set of "environmental problems"; for these people the "environment" becomes rather a means to promote the "growth" of the economy: "let us invest in 'green technologies' and GDP will start to grow again". It has barely to be said that the "environment" has no problem at all; 


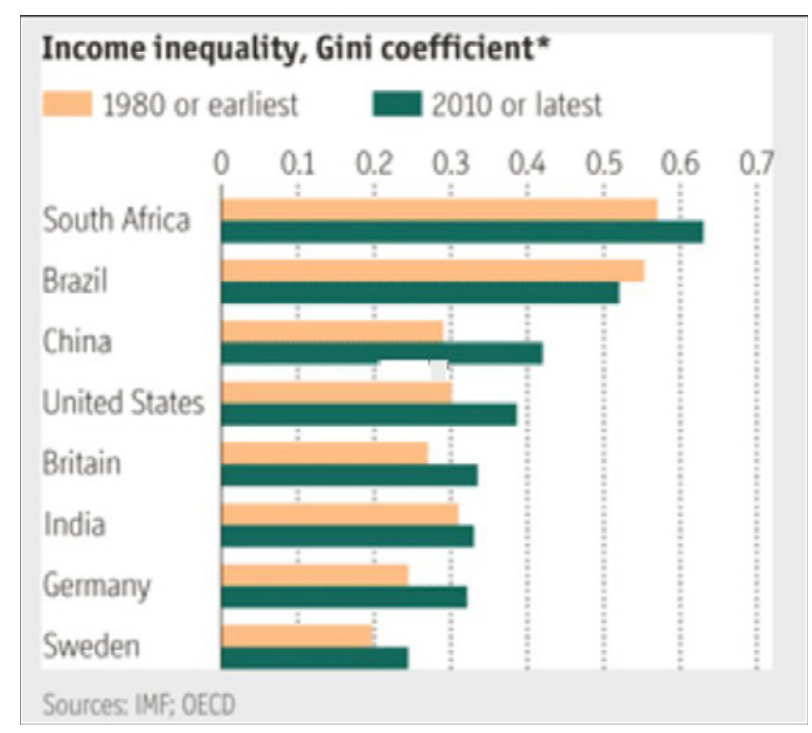

Figure 2. Time evolution of the Gini coefficient in the world. The Gini coefficient measures the inequality of the income distribution. The situation has worsened almost everywhere in 30 years; Brasil, where the inequality is very high, is an exception.

rather it is we only who have problems. This attitude has the rationality of a guy with a broken leg who plans to participate to a high jump competition.

The second option implies an always present debate on the closeness or not of the walls that prevent an indefinite "growth". We are going to discuss this matter tomorrow, but I think I can anticipate that by now the limits are indeed very close, even on the time scale typical of human affairs. In any case recognizing the problem, then differing it, for sure does not solve it. It recalls a bit the sentence "In the long run we are all dead" by John Maynard Keynes, or the "après nous le déluge" by Louis $\mathrm{XV}$ of France, or even the "seize the day", modern version of Horace's carpe diem. Rationality dwells elsewhere.

Coming to the third solution some might propose, maybe we will discuss it in the next days. There are however various inconveniences. In order to allow GDP to grow keeping the amount of manipulated matter fixed, one must let the number of "manipulations" performed on a given amount of matter increase, but also the "manipulations" unavoidably have a material (in particular energy) content which is subject to the same constraints as the whole material world. Furthermore there is a more subtle limit: if we want the volume of exchanges to grow keeping the exchanged quantities fixed, we must increase the exchange velocity, but velocity too has an insurmountable upper limit in any given physical system. I'll come again on this later. One might think to only build on virtual exchanges, like the more and more happens in the world of finance, but even so a remark is inescapable: if quantities remain stable but the swirling exchange of "access titles" grows, what grows in the end, like in a worldwide Monopoly game, are inequalities. There is evidence of this phenomenon in our world, even though, at the moment one pretends quantities also to go on growing: there is a limited number of subjects, in prospect the more and more limited, who own disproportionate "withdrawal rights" against a growing number of subjects who are losing their economic autonomy; see figure (2). Of course this is the type of growth that produces more immediately explosive effects. 


\section{An example: governing an ever-growing system}

Mine is an introductory relation, so that I limit this talk to a review of various problems and topics without examining them in depth. Thorough analyzes will be made in the course of the conference. Nonetheless, before closing, I would like to develop an exercise, touching upon features of the "growth" mechanism, which are usually not considered or remain in the shade.

Everybody knows that there is a problem with, and a live debate on, anthropogenic climate changes; that there is a problem with the depletion of energy resources and with the scarcity of mineral resources; that there is a problem with fresh and clean water; degradation and reduction of available farming soils is an issue often pointed out to the public, and so on. About these topics we shall discuss in the next days.

Now I would like to draw your attention on the control and safety of a growing complex system. I will do it in a simplified way trying to reduce as much as possible the technicalities and to evidence the fundamental machinery.

\subsection{A network of exchange links}

An economy, as we have seen, is based on a system of exchange relations. The growth that many invoke implies also an increase of the exchange opportunities, the number of companies, the market segments. We may think of human beings exchanging something among them; or villages connected by pathways; or factories and markets connected by roads and information channels.... We may sketch all this thinking of a three-dimensional network (I should say a graph) whose vertices (or knots) are the things I have just mentioned. Let us suppose that the vertices are in the number of $N$. The produced wealth is not per se depending on the number of vertices, but rather on the exchange fluxes among them and, in an abstract market, all knots should be on the same footing. In other words the best conditions for such economy would be obtained if any single knot could exchange something with any other single knot in the network. The relevant quantity emerging from these considerations is the number of links among the knots. Now, the total number $\mathfrak{N}$ of possible links among $N$ vertices is given by a simple formula of the binomial development:

$$
\mathfrak{N}=\frac{N(N-1)}{2}
$$

You may notice that when $N$ grows, $\mathfrak{N}$ grows faster and precisely with a quadratic law: to 2 vertices it corresponds 1 possible link; 3 vertices, 3 links; 4 vertices, 6 links; 5 vertices, 10 links; and so on.

So far I have spoken of possible links, but wealth originates from actual exchanges, not from possible exchanges. Once a channel is available, the flux through it will initially be small, but, if wealth, whatever it is, depends on the total flux and people wants it to grow, then also the flux on each single link must grow. If we call $n_{i}$ the number of transactions or exchanges or trips per year through the $i$-th available connection, the total annual volume of exchanges $\Phi$ will of course be

$$
\Phi=\sum_{i=1}^{\mathfrak{N}} n_{i}
$$

The formula may be simplified a bit using the average annual flux through a link, $\langle n\rangle$, and becomes

$$
\Phi=<n>\mathfrak{N}
$$


If I wouldn't be afraid of the economists' reproaches I would be tempted of saying that $\Phi$ is proportional to the Gross Domestic Product of our system; anyway I shall say that it is proportional to the wealth $R$ produced by the system:

$$
R=\rho \Phi
$$

The $\rho$ factor is a parameter carrying all the ambiguity of quantities measured by money, however if $R$ is bound to grow, as it is maintained by many, the same will do $\Phi$ and this will be possible either through any of the two factors in formula (2) or through both jointly. In any case the growth of the number of possible links, $\mathfrak{N}$, requires the growth of the number of "poles" $N$.

Coming to the average annual flux through a link, in its evaluation also those links enter which are in principle accessible but not operating and along which the flux is zero; this fact implies that $\langle n\rangle$ can grow: a) letting the number of active exchange links increase up to the greatest possible value for a given number of vertices [formula (1)]; b) letting the flux through each single channel grow. Now, option a) has to comply with the maximal capacity of a single vertex, which in turn depends on the nature of the vertex: if we have to do with an electronic communication network the knots are electronic devices (servers, routers, laptops...) each of which has a maximal capability of managing separate connections, because of the physical constraints typical of the employed materials, of the commutation speed, etc. In order to avoid a typical objection of those who deny the existence of limits, I immediately specify that I am not referring to specific machines of the present technological generation that would then be surpassed by the next generation, then by further one, then.... . I am referring to ideal machines working only on the base of the physical principles which rule the atomic physics (the branch of physics at the base of the applications of electronics) and invariably imply the saturation of the capacity of any calculation or data processing device.

If the vertices of the graph are human beings the problems are the same but for the fact that saturation is reached much earlier.

Let's come to option b). Growth requires an increase of the flux transiting over every single link of the network. Here I will not spend too many words: be it a road or an optical fiber, a railway or a satellite communication channel, under a continuous push towards growth the trend in time of the flux is represented by a logistic curve, like the one shown in figure (3)

The saturation of all or part of the links is unavoidable and the saturation level depends on the nature of the link. Under a continuous pressure towards growth the links tend to saturate and consequently push towards the saturation of the number of links manageable by every single vertex. Finally the growth must necessarily end being transferred on the global size of the network, i.e. to the number of vertices. Applying all this to the whole human kind it turns out that the "growth" must in the end be demographic also; if the number of "vertices" of the graph does not grow and "wealth" must grow, the system saturates and jams. On the other hand the growth of the number of vertices has to comply with the physical constraints of the environment where the growth takes place. The debate could go on and evolve for long, but would not be per se new: after all I have just presented in a more abstract way the subject of "The limits to growth" and in the next days various specific lectures will do better than I do. However the aspect I want to highlight in the behaviour of our network is not the one of the physical constraints.

\subsection{The problem of safety/reliability}

Let us come again to the number of exchanges that happen per year on the average through a link of our network, $\langle n>$; each exchange, if it is real, is unavoidably associated with a probability, 


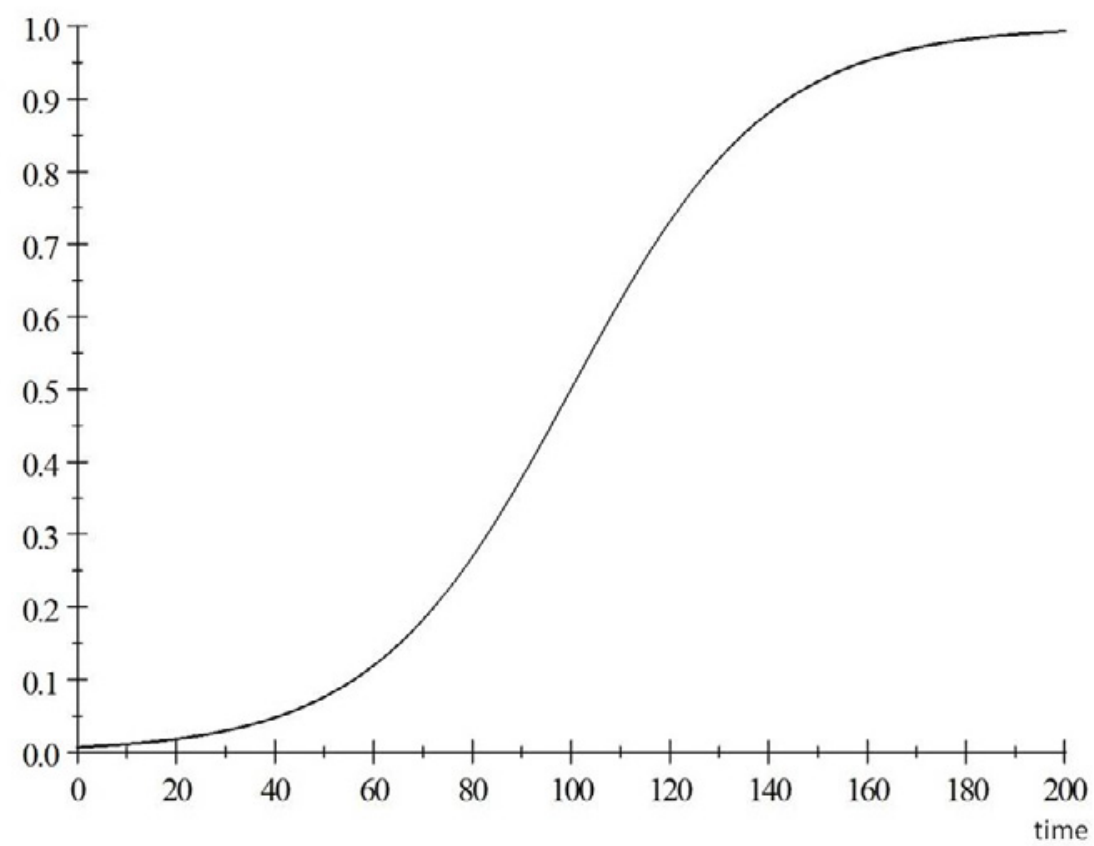

Figure 3. A typical logistic curve describing the saturation in time of a channel in which a continuous thrust towards the increase of the flux is present.

$\varepsilon$, of undergoing some inconvenience, breakdown, accident, malfunction. Over big numbers this probability becomes statistically a global real annual number of "inconveniences", $v$, given by

$$
v=<\varepsilon><n>\mathfrak{N}=<\varepsilon>\Phi
$$

If we have considered $\Phi$ as being proportional to the gross "wealth" produced during the year, we may think $v$ being proportional to the damage $D$ overall suffered because of the happened inconveniences:

$$
D=\rho v
$$

Strictly speaking damage is not simply proportional to the gross wealth: in general $\langle\varepsilon\rangle$ (which is contained in $v$ ) depends in turn on $\Phi$. Let us think for instance of a road with vehicles travelling on it; keeping technology, road and driver fixed, the probability of individual accident grows with the flux of vehicles.

Summing up, our system behaves more or less like a ferromagnet: magnetization grows more than proportionally with the intensity of the magnetizing field and tends sooner or later to reach a fixed saturation value. The dependence of $\varepsilon$ on $\Phi$ changes according to the type of "inconvenience" and sometimes it may also be absent: let us merge all this into a hypothetical average of all averages in order to arrive to the $\langle\varepsilon\rangle$ of (4), that will now represent the total "risk". In general, and when we are far from saturation, we expect insecurity to grow while wealth grows, i.e. we expect the ratio between risk and gross benefit to worsen. Of course this is an unwanted and not at all relished effect; 


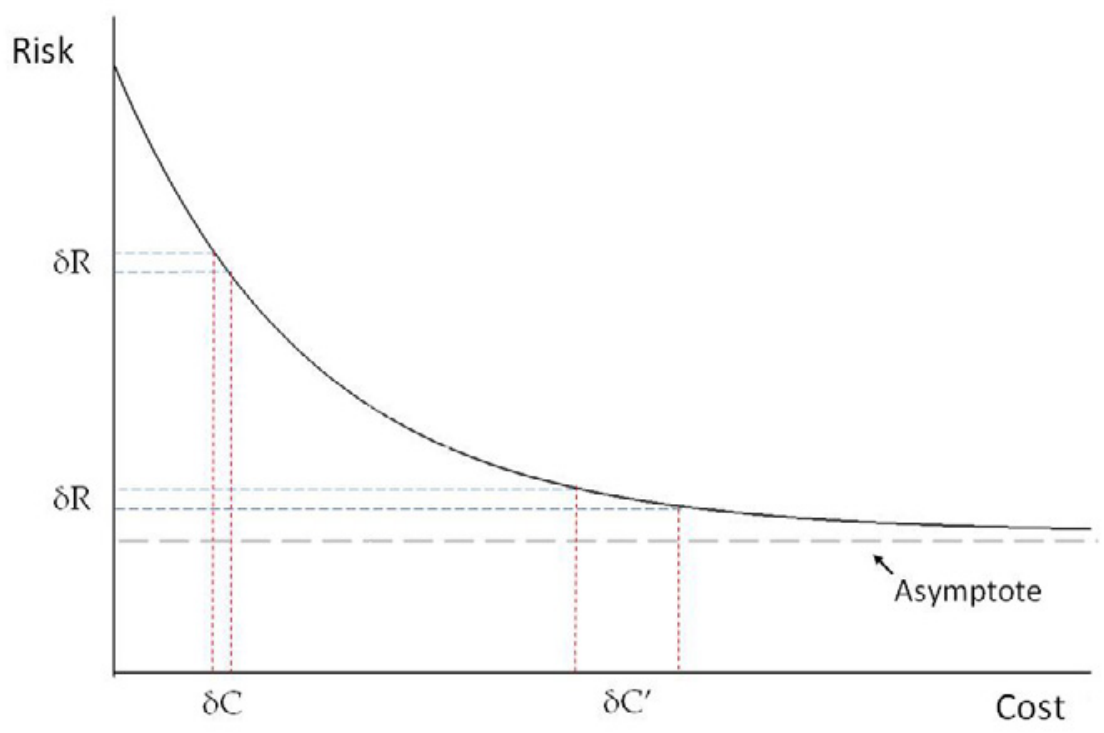

Figure 4. Asymptotic decrease of "risk" with increasing cost. The same $\delta R$ corresponds to different $\delta C$

is it possible to nullify it? Be it car crashes, engine breakdowns, noise injected into a communication channel, robberies, frauds, bribery or else, one may hope to keep everything under control below a previously fixed threshold, by means of an improvement of the efficiency of the system, i.e. of the technologies, the methods and the controls. And here the problem of the cost of all that comes to light.

The efficiency of a system, interpreted as the ratio between desired effects and globally produced effects, cannot grow indefinitely. It stands to reason that the maximal abstractly attainable value would be 1; actually even the maximal value attainable in principle is less than 1: "side unwanted effects" are necessary, whatever the quality of the employed technology is. Engineers and physicists in the audience have undoubtedly realized that I am speaking of the first and second principles of thermodynamics: that kind of laws that parliaments do not vote and cannot amend, on which autocrats have no power, which are totally unaffected by the subtleties of rhetoricians.

Consistently with the example I am trying to work out, rather than to efficiency I will refer to the probability of "inconvenience" for each single exchange: let us say that we will keep an eye on risk. Since $\langle\varepsilon>$ grows more than proportionally with the flux $\Phi$ in the network, we shall try to compensate the unbalance reducing the risk for a given flux. This aim, as I have already said, can be pursued improving "efficiency" by various technological, behavioural, regulatory measures. All this has a cost, no matter how you evaluate it, and the typical trend of risk as a function of "cost" is schematically shown in figure (4).

What happens is well known: at the beginning, when the efficiency is low and the risk is high, one obtains relevant results with limited cost, but once the risk is already relatively low further improve- 
ments have bigger and bigger costs. Let us swap the axes in the figure (or rotate it by $90^{\circ}$ on the left): one clearly sees that when the "risk" tends to the asymptote the cost diverges. Of course the cost that we can call 'of security' must be subtracted from the gross wealth $R$, proportional to $\Phi$, so that the actually available wealth, $\mathcal{U}$, decreases.

So far the colloquial and qualitative description of what happens. Let us now try a numerical exercise. Start from the gross wealth $R$. According to the wishes and also to the "doctrine" $R$ (if you prefer: the GDP) should steadily grow with a geometric progression; in practice its evolution in time should be described by an exponential function:

$$
R=R_{0} e^{\chi t}
$$

We should choose a value for $\chi$. Initially I thought of $2 \%$ per year. However, it occurred to me of reading, on the newspaper La Stampa of September 5 this year, an article by a professor of economics of the Turin University, Prof. Mario Deaglio, inspired by some sentences of a singer (Jovanotti) about the "wonderful" (environmentally friendly) growth. Prof. Deaglio writes ${ }^{1}$ "a 1 per cent growth per year has nothing «wonderful», a 5 per cent growth would be unattainable. Jovanotti's «Wonderful growth» corresponds to an average annual increase of the gross domestic product (the notorious GDP) in the order of a long term 2.5-3 per cent. Such growth rate has to be steady, nondehumanizing, not particularly consumeristic." That growth rate, according to Deaglio, would include both the productivity and the employment increase; no material quantity is mentioned: no tons, no kilowatt hour, no mundane variable that could disturb the "wonderful growth". In line with this approach, I will then choose

$$
\chi=0.03 / \text { year }
$$

which of course corresponds to a similar growth rate for the exchange volume $\Phi$.

We must now deal with (4) and (5). The majority will expect the total risk to remain constant or even to decrease while the produced wealth increases; hence we deduce that people will act upon what I'll generically call the technology in order to try and obtain a decrease of $\langle\varepsilon\rangle$ in time big enough to compensate for the growth of $\Phi$, i.e. people will require that

$$
<\varepsilon>=\varepsilon_{0} e^{-\chi t}
$$

Strictly speaking this result is impossible: no real transfer may happen at zero risk. Let us however neglect this remark and let us pass to consider the total cost of security $C$, that is the quantity schematically represented on the abscissae of fig. (4). The trend depicted there may be expressed by the formula:

$$
C=\frac{\kappa}{\varepsilon-\varepsilon_{*}} \Phi=\frac{\kappa}{<\varepsilon>-\varepsilon_{*}} \frac{R}{\rho}
$$

or even by a number of more complicated expressions. This is just an exercise, so let us stay happy with (9) where one sees both the proportionality to the total exchange flux, then in practice to the produced wealth, and the divergence in correspondence of the asymptotic value $\varepsilon_{*}$. Let us imagine to start from a situation at time 0 where the cost of safety is a fraction $\psi$ of the total wealth, so that we may write:

$$
C=\psi \frac{\varepsilon_{0}-\varepsilon_{*}}{<\varepsilon>-\varepsilon_{*}} R
$$

\footnotetext{
${ }^{1}$ The English translation is by the author of the present Introduction.
} 


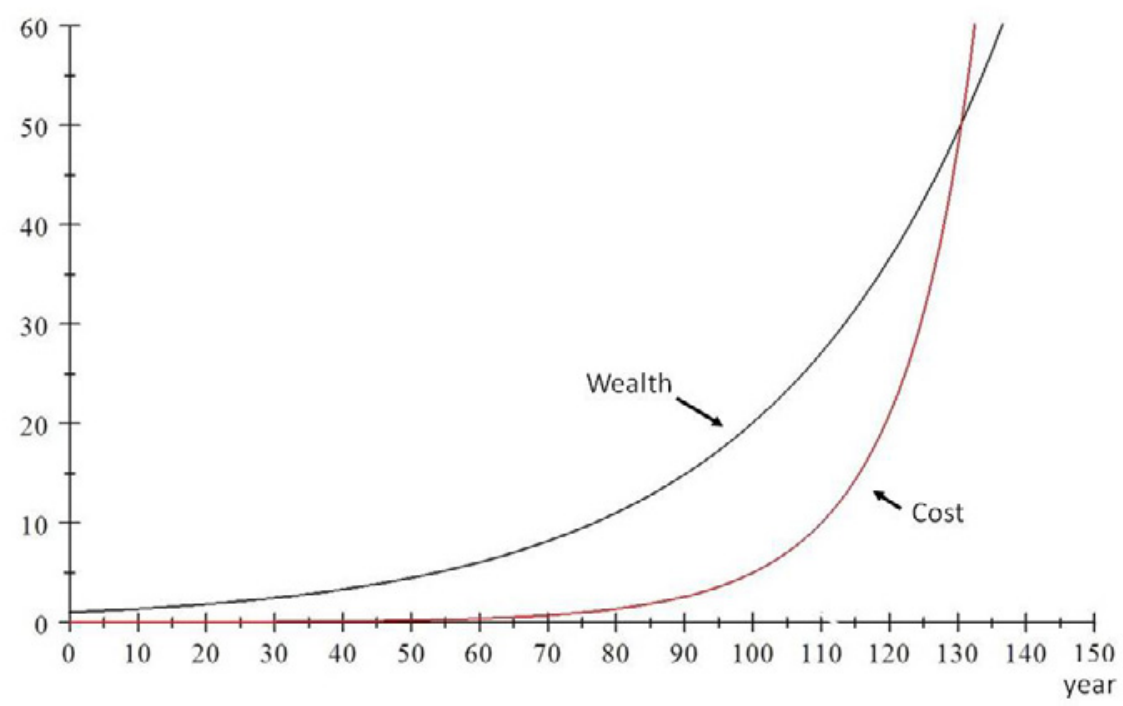

Figure 5. The black curve represents the growth of the gross wealth at a rate of 3\% per year. The red curve is the time trend of the safety cost, starting from a 1 to 100 ratio with respect to the asymptotic value and of the initial cost with respect to the initial wealth.

Making the time dependences explicit, the formula becomes

$$
C=\psi \frac{\varepsilon_{0}-\varepsilon_{*}}{\varepsilon_{0} e^{-\chi t}-\varepsilon_{*}} R_{0} e^{\chi t}
$$

Let us try and see what happens if the initial cost is $1 \%$ of the initial wealth and the initial risk is 100 times bigger than the asymptotic value. Figure (5) shows the time evolution both of $R / R_{0}$ and of $C / R_{0}$ :

The most interesting portrayal is obtained when graphing the difference, $\mathcal{U}$, between wealth and safety cost, normalized to the initial wealth; see figure (6).

Looking at the diagram a quotation from Leonardo da Vinci comes spontaneously to my mind:

Chi scalza il muro, quello gli cade addosso. ${ }^{2}$

During about 115 years the net wealth or profit, $\mathcal{U}$, grows but in the end it plunges catastrophically in more or less fifteen years. Of course the result depends on the numerical values I have chosen. Keeping the "wonderful" 3\% fixed, what is the change if I modify the ratio between the initial and the asymptotic risk? Let's see.

Even assuming a $1000: 1$ ratio between initial and asymptotic risk, the trend remains the same and the "gain" in time is around twenty years. If instead the annual growth rate is limited to $2 \%$ the gained years are about sixty.

\footnotetext{
2 "If you dig up the wall, it will fall upon you". Free translation by the author.
} 


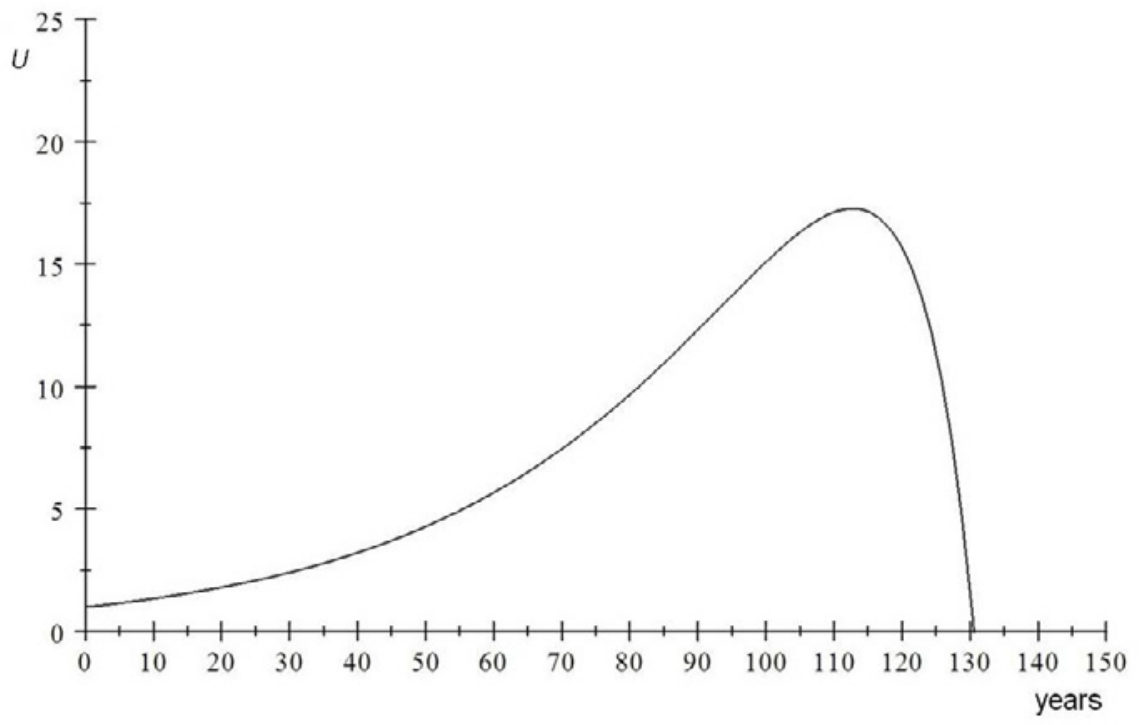

Figure 6. Time evolution of the net wealth given by the difference between the gross wealth produced by the growing system and the cost of safety.

This is just an exercise. The real world is far more complicated than that, but ...

A trend like the one depicted in fig. (6) is often found in the history of human societies, from the Sumerian civilization to the Roman empire, to many other cases. The example of the Sumerians is especially interesting because, in their case, the collapse was due to the unforeseen (and unforeseeable at the time) side effects of a great technical advance. The development of a sophisticated irrigation system in a dry climate brought, because of evaporation, to irreversibly salt the soils and led their productivity to collapse. The productivity of the former Sumerian land remains quite low even today, after four thousand years.

The typical feature is a blooming, almost triumphal, raise followed by a much shorter and steeper descent:

...the raise is gradual, the ruin is precipitous... (Lucius Anneus Seneca, Letters to Lucilius, 91-6 ${ }^{3}$ ).

We could call it, as Ugo Bardi does, "Seneca effect". For completeness I should mention that he also introduces a "Cassandra effect" that we would like to avoid here.

Besides the historical examples are there circumscribed phenomena where mechanisms are operating like the ones I described above? Let us think to the role (and cost) of bureaucracy in our society. Let us think to the development of e-mail networks and to the percent of our work time we must dedicate to the handling of the correspondence: each communication is very quick, the answer time is

${ }^{3}$... incrementa lente exeunt, festinatur in damnum. 


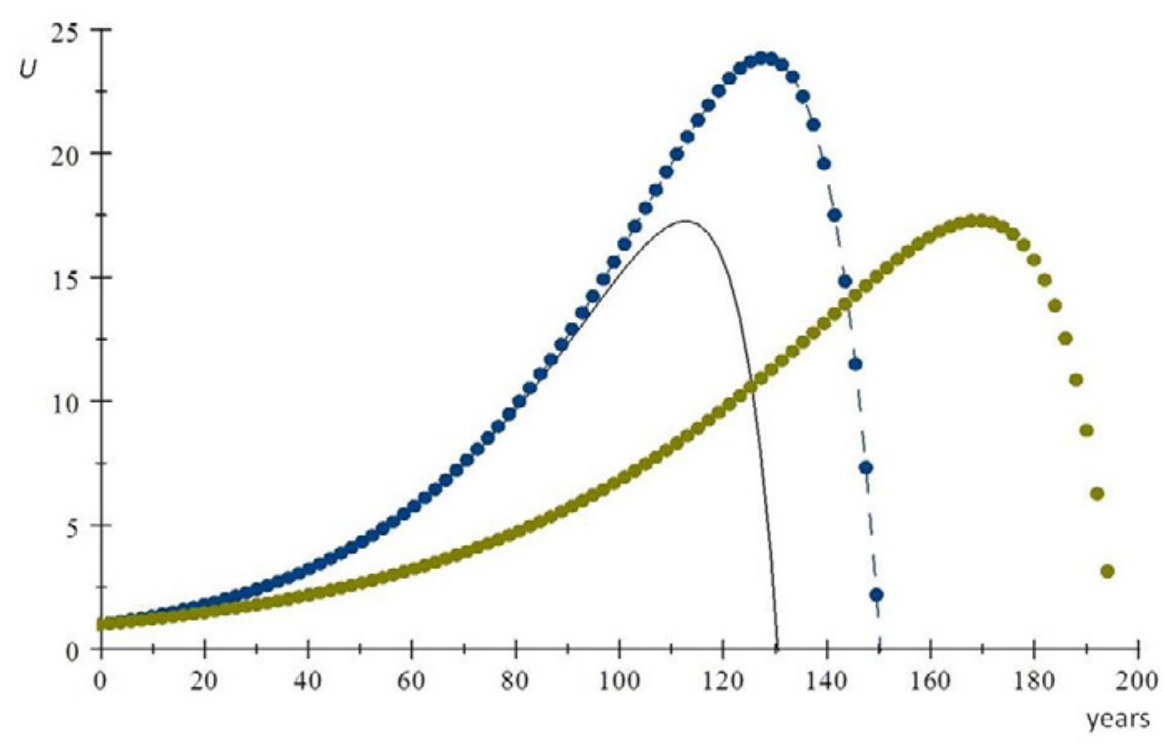

Figure 7. Black curve: reference case. Blue curve: $\varepsilon_{0} / \varepsilon_{*}=1000$. Greenish line: $\chi=0.02$.

not. Let us think to informatization and to the queues before the counters of public offices: the length of the queue does not decrease, the number of counters does.

\subsection{Saturation and capacity of keeping processes under control}

In our idealized network of exchange fluxes I have pretended to believe that each single vertex can in principle be connected with any other vertex of the net: this hypothesis is the base of (1). However, as we have seen, both the exchange channels and the vertices get saturated, which fact leads to transferring the growth to the total number of vertices. Unavoidably then it becomes gradually impossible for a vertex, even in principle, to directly communicate with all other vertices. This fact does not prevent the communication with the parts of the net out of reach, but implies that the related flux be mediated by the vertices with which the link is direct; the indirect flux must however be transmitted through the vertices with which one is in direct touch. Every vertex, then, must support its own exchange flux together with the flux in transit. The latter then shortens the saturation times of any single vertex. In other words we can say that a competition is established concerning the allocation of time to control your flux against the flux in transit; otherwise stated, the more are the vertices used for simple transit, the slower becomes the distant exchange. Summing up, the myth of globalization must face the problem of control: the growth of the network implies both a parceling of the system in governable islands and a functional hierarchy of the vertices.

A complicated way to say that an upper limit exists to the physical size of a single living being composed of cells: there have never existed multicellular animals bigger than the blue whale (maybe 
equalled, but not surpassed, by some terrestrial dinosaur of the Jurassic period). With a growing size the system goes out of control by a single will.

So far I have exorcised a word I could and maybe I should have used: entropy. It is a physical quantity introduced by thermodynamics, that can easily be misused. Anyway, the popular version of its definition says that it is something having to do with disorder in a system made of a very big number of components; and this is true. Furthermore one of the non-negotiable laws asserts that in an isolated complex system whatever happens forces entropy, then disorder, to grow. The surface layer of our planet is not an isolated system, but it exchanges with the external space almost only radiation. If the number of physical processes taking place annually on earth remains more or less stable, so does the entropy and this is because it is possible to expel energy to space. However if the number of thermodynamic transformations increases, then, in order to keep the entropy stable, the surface temperature of the planet must grow. The phenomenon about which I am speaking now is not the world famous green-house effect, on which the discussion will be tomorrow: rather it is an additional complication.

So far the most traditional meaning. It is however possible to define a statistical entropy associated to complex systems irrespective of strictly thermal variables. Order and disorder of the books on a shelf can be evaluated in terms of entropy and it is true that, without "external" interventions for reordering, the more books you draw and use the more disorder grows. Restoring or maintaining order requires a dedicated work, that can be assessed in terms of time (to be subtracted from the productive time) then finally in terms of money. We come back, by another way, to the Seneca effect.

\section{Treating the addict}

I have touched upon a number of problems and effects, that could be analyzed in more formal and technical terms. The relevant feature, however, is that they are not debatable, even though there are many uncertainties in their formal description and in their parametrization.

Well, no discussion nor, least of all, any account is taken of them in the sites where decisions relevant for the community are taken; excepting, at most, fortuitous actions on symptoms when acute effects appear.

A comparison that comes to my mind is with a doctoral team facing an addict or an alcoholic in a drug withdrawal syndrome. The patient is sick, very sick, because he cannot find the dose. Suffering is real and the danger of irreversible damages is real too. Everybody knows that, in this situation, the sheer block of the assumption of the dependence giving substance is not viable; it is necessary to define a proper exit strategy that includes a gradual elimination of the substance, accompanied by a controlled administration of some less toxic substitute and by protective and healing measures. In any case the final goal is to free the patient from his dependence.

However in the case of the "growth" disease the medical team, up against recurring withdrawal crises, has only one strategy: to strive for providing the patient with the dose he misses and, since the dependence increases more and more, to try and procure in turn increasing doses. The final result of such a "therapy" is rather evident.

The fact that makes things harder is however that in our case the patient and the medical team essentially coincide.

Those who professionally deal or have the misfortune to care alcoholics or drug addicts perfectly know one thing. That any exit strategy from a dependence state by means of a withdrawal therapy requires an inescapable preliminary condition: the patient must agree. And this is the mess. 


\section{Reason or scope intelligence?}

Human beings have a bizarre feature: their mind is equipped with analysis tools of the reality which enable, in a reasonably reliable way, to forecast the consequences of their behaviours even over medium/long times and over distances much bigger than the ones they can directly inspect by means of their senses. Let us call this ability: reason.

Human beings however, to some extent, share with the other animals an apparently similar ability, which however does not bother about the future, if not immediate, and about the space range, if not directly perceived by the senses. This ability I could call "scope intelligence".

We know that chimpanzees are able to build simple tools, in order, for instance, to facilitate the extraction of honey from honeycombs hosted within holes in the logs of trees. Crows too have learnt, in order to smash nut shells, to bring them flying at a given height then letting them fall against a hard surface. Examples are abundant and describe the nature of what I have called scope intelligence.

Let us now imagine some chimpanzees who, keeping the purpose to pull out honey from logs (or to procure everyday's food in similar situations) learn to build not only simple wooden sticks to be introduced into the cavity then licked, but drills, diggers, explosives and so on. This peculiar species of "advanced" chimpanzees would rapidly reach the depletion of the food resources of its environment, ending then in its own extinction.

Specific human civilizations have indeed concluded their life like that, collapsing under the unforeseen effects of some progress introduced thanks to the scope intelligence and in the helplessness of reason: I have already mentioned the example of Sumerians and the salinization of their agricultural land, source of their prosperity.

Today's situation, however, is still different, because reason is now able to predict many effects of an expansion of economy that pretends to last indefinitely; but this type of prediction is either ignored or rejected. We are facing a real conflict between reason and scope intelligence, between human and animal nature, and the match risks to be compromised by a fact ironically expressed by Albert Einstein who happened to remark that

\section{the difference between stupidity and genius is that genius has its limits.}

Notwithstanding the difficulties, however, most of the international scientific community believes that we can make the grade and that reason will in the end have the upper hand on the scope intelligence.

Our condition has very well been described in what we could call a laic parable told in the 19th century by Robert Louis Stevenson: Strange case of Dr Jekyll and Mr Hide. Dr Jekyll is a reasonable and respectable person, he is aware of what is wise to do; however the man who takes decisions on what to do is Mr Hide. One might think, and very often some think so, that it would suffice for Jekyll to get rid of Hide and everything would be solved, but when one tries that way tragically realizes that Dr Jekyll and Mr Hide are the same person...

This is a conference for Dr Jekyll, who assembled here in order to clarify his mind on what to do. The point however is that afterwards it will be necessary to bridle Mr Hide. We are going to tackle this problem again at the end of our four days. 